\title{
Hepcidin Level Changes in Type 2 Diabetes
}

\author{
FOUAD T.F. ATYIA, M.Sc.*; AMR M. GAWALY, M.D.; ENAAM S. ABD EL-BAR, M.D.** and \\ ABD EL-MOTELB T. EISSA, M.D.* \\ The Departments of Internal Medicine* and Clinical Pathology**, Faculty of Medicine, Tanta University
}

\begin{abstract}
Background: Diabetes mellitus is a group of metabolic diseases characterized by hyperglycemia resulting from defects in insulin secretion or insulin action, or both. Diabetes and its complications have become a major public health problem in the world and its prevention has become a public health priority. Hepcidin, a 25 -amino-acid antimicrobial peptide, is the central regulator of iron homeostasis. Under normal circumstances, hepcidin expression and subsequent release into plasma prevents further absorption of iron from the duodenal enterocytes by preventing the efflux of iron by ferroportin channels, hence reduced amounts of iron delivery via transferrin to hepatocytes. In response to iron loading, hepcidin expression increased to prevent the further uptake of iron. Conversely, during iron deficiency, hepcidin expression decreased.
\end{abstract}

Aim of the Study: Was to assess the possible changes of serum hepcidin that may occur in patients with type 2 diabetes.

Objectives: Was to evaluate changes of serum hepcidin level in type 2 diabetes, assess possible relationships of serum hepcidin, iron status, hepcidin: Ferritin ratio and HOMA-IR in type 2 diabetes patients.

Methods: This study consisted of randomized eighty subjects divided into four groups: Group 1: Included 20 patients with impaired glucose tolerance (pre-diabetes), Group 2: Included 20 patients with controlled diabetes, Group 3: 20 patients with uncontrolled diabetes, Group 4: Included 20 healthy volunteers.

Results: Hepcidin: Ferritin ratio was statistically high in impaired glucose tolerance and low in uncontrolled diabetes with ( $p$-value ${ }^{\left.<0.001^{*}\right)}$ and normal in controlled diabetes and healthy volunteers. Significant negative correlation between hepcidin: ferritin ratio and HOMA-IR in impaired glucose tolerance with $(p$-value $=0.009 *$ ) was found.

Conclusion: Serum hepcidin affected by multiple factors so cannot be used for screening of type 2 diabetes. But hepcidin: Ferritin ratio could be a novel marker for early screening of patients with type 2 diabetes.

Key Words: Hepcidin - Diabetes - Iron.

Correspondence to: Dr. Fouad T.F. Atyia, The Department of Internal Medicine, Faculty of Medicine, Tanta University

\section{Introduction}

EVIDENCE suggests that iron plays a role in the pathogenesis of Type 2 diabetes mellitus. Iron influences glucose metabolism, even in the absence of significant iron overload. Mildly elevated body iron stores are associated with increased fasting serum insulin and blood glucose. Lowering iron stores by venesection increases peripheral insulin sensitivity in patients with high-ferritin Type 2 diabetes [1]

Hepcidin is the key hormone regulating iron homeostasis. It is a 25 -amino-acid peptide predominantly synthesized in the liver. Hepatic secretion of hepcidin in response to iron overload negatively regulates iron homeostasis. Hepcidin prevents iron efflux from enterocytes, macrophages and hepatocytes into the plasma by inducing internalization and degradation of the iron exporter ferroportin in these cells [2].

The underlying mechanism for the increased body iron Stores in conditions of insulin resistance is unclear. The Hepcidin: Ferritin ratio has been used as a marker of the adequacy of hepcidin production for a given iron load. We investigated whether insulin resistance is related to a decrease in hepcidin: Ferritin ratio, and thus to inadequate hepcidin levels. Understanding the mechanism underpinning the iron overload associated with insulin resistance may help develop novel therapies for Type 2 diabetes [3].

The relationship between iron metabolism and insulin resistance is bidirectional, as iron accumulation favours insulin resistance and insulin resistance may inturn result in increased body iron stores [4]. 


\section{Patients and Methods}

This study was conducted on 80 subjects with type 2 diabetes (44 males and 36 females) admitted to inpatient wards and outpatient clinic of Internal Medicine Department of Tanta University Hospital between January 2016 and June 2017 and 20 healthy age and gender matched volunteers were enrolled as the control group. All participant provided informed written consent and the study was approved by Tanta Faculty of Medicine Ethical Committee.

The participants were divided into the following groups:

Group (1): 20 patients with impaired glucose tolerance (prediabetes).

Group (2): 20 patients with controlled type 2 diabetes.

Group (3): 20 patients with uncontrolled type 2 diabetes.

Group (4): 20 apparently healthy volunteers of matched age and sex will be recruited as controls.

\section{Exclusion criteria:}

Patients with anemia, renal insufficiency, autoimmune diseases, chronic liver diseases, chronic infection, malignancies, chronic medical illness, psychiatric illness, alcohol abuse.

\section{Inclusion criteria:}

Patients with uncontrolled type 2 diabetes, controlled type 2 diabetes and impaired glucose tolerance.

\section{Method:}

All participants in this study were subjected to: Thorough history taking, full clinical examination, laboratory investigations in the form of: Complete blood count, $\mathrm{HbA} 1 \mathrm{c}$, fasting \& $2 \mathrm{hrs}$ post prandial blood glucose, lipid profile (serum cholesterol \& triglyceride), serum uric acid, serum creatinine, serum hepcidin, HOMA-IR, serum ferritin, total iron binding capacity (TIBC), serum iron, transferrin saturation (TSAT).

\section{Sampling and laboratory investigations:}

Sampling and all laboratory investigations were done in Clinical Pathology Department, Tanta University Hospitals.

Full Blood Count: Was performed using BCC3000 Auto Hematology Analyzer.
Serum hepcidin using enzyme-linked immunosorbent assay (ELIZA).

HOMA-IR: Insulin ( U/ul) measured using radioimmunoassay kits Pharmacia and plasma glucose $(\mathrm{mmol} / \mathrm{L})$ measured by a glucose oxidase method fasting blood glucose from venous blood (B-Gluc) and converted to plasma glucose value (correction factor 1.13).

Serum ferritin, total iron binding capacity (TI$\mathrm{BC}$ ), serum iron, transferrin saturation (TSAT): Was performed using kits for measuring serum iron and TIBC (Stanbio Iron and Total Iron Binding Capacity, Texas).

\section{Statistical analysis of the data:}

Data were fed to the computer and analyzed using IBM SPSS software package version 20.0. Quantitative data were described using range (minimum and maximum), mean, standard deviation and median. Comparisons between more than two populations were analyzed using F-test (ANOVA). ROC-curve was used for assessment of sensitivity and specificity. Significance of the obtained results was judged at the $p$-value $<0.05$.

\section{Results}

Table (1) showed that there was no statistically significant difference between four studied groups as regard age and S. Iron $(\mathrm{Ug} / \mathrm{ml})$ and there was statistically highly significant between studied groups as regard fasting blood sugar (FBS), 2hrs post prandial blood sugar (PBS), HbA1C, HOMAIR, Hepcidin, serum ferritin, transferrin saturation (T.Sat), TIBC and Hepcidin: Ferritin ratio.

Fig. (1) showed that hepcidin was statistically highly significant between studied groups and between group $1 \&$ group 2 and group $1 \&$ group 3 and group $1 \&$ group 4 and group $2 \&$ group 3 and group $3 \&$ group 4 with ( $p$-value $<0.001 *)$.

Fig. (2) showed that hepcidin: Ferritin ratio was statistically highly significant between studied groups and between group $1 \&$ group 2 and group $1 \&$ group 3 and group $1 \&$ group 4 and group 2 $\&$ group 3 and group $3 \&$ group 4 with ( $p$-value $<0.001 *)$. 
Table (1): Comparison between studied groups regarding age, FBS, 2hrs PPS, HbA1C, HOMAIR, Hepcidin, S. ferritin, T.Sat, S.iron, TIBC and Hepcidin ferritin ratio.

\begin{tabular}{|c|c|c|c|c|}
\hline & Range & Mean \pm S. D & $F$. test & $p$-value \\
\hline \multicolumn{5}{|l|}{ Age: } \\
\hline Group 1 & $41-67$ & $49.25 \pm 6.15$ & 0.186 & 0.905 \\
\hline Group 2 & $39-63$ & $49.85 \pm 5.74$ & & \\
\hline Group 3 & $40-62$ & $48.60 \pm 5.58$ & & \\
\hline Group 4 & $40-65$ & $48.55 \pm 7.76$ & & \\
\hline \multicolumn{5}{|c|}{$F B S(m g / d l):$} \\
\hline Group 1 & $110-125$ & $117.65 \pm 3.83$ & 167.425 & $0.001 *$ \\
\hline Group 2 & $96-144$ & $125.30 \pm 14.72$ & & \\
\hline Group 3 & $243-482$ & $324.45 \pm 73.29$ & & \\
\hline Group 4 & $77-96$ & $86.80 \pm 5.24$ & & \\
\hline \multicolumn{5}{|c|}{$2 h r s$ PBS $(m g / d l)$ : } \\
\hline Group 1 & $150-188$ & $165.85 \pm 9.17$ & 392.724 & $0.001 *$ \\
\hline Group 2 & $150-191$ & $173.40 \pm 10.93$ & & \\
\hline Group 3 & $334-572$ & $425.65 \pm 59.76$ & & \\
\hline Group 4 & $111-139$ & $124.75 \pm 7.58$ & & \\
\hline \multicolumn{5}{|l|}{$\operatorname{HbAlC}(\%):$} \\
\hline Group 1 & $5.5-6.5$ & $5.97 \pm 0.25$ & 600.860 & $0.001 *$ \\
\hline Group 2 & $6.2-8$ & $6.80 \pm 0.38$ & & \\
\hline Group 3 & $8.7-10.6$ & $9.57 \pm 0.54$ & & \\
\hline Group 4 & $4.1-5$ & $4.69 \pm 0.27$ & & \\
\hline \multicolumn{5}{|l|}{ HOMA IR: } \\
\hline Group 1 & $0.3-1$ & $0.75 \pm 0.19$ & 4.391 & $0.007 *$ \\
\hline Group 2 & $1.3-3.5$ & $2.34 \pm 0.83$ & & \\
\hline Group 3 & $1.7-771$ & $80.46 \pm 168.92$ & & \\
\hline Group 4 & $0.7-1$ & $0.89 \pm 0.10$ & & \\
\hline \multicolumn{5}{|c|}{ Hepcidin $(n g / m l):$} \\
\hline Group 1 & $257-370$ & $303.35 \pm 29.45$ & 98.644 & $0.001 *$ \\
\hline Group 2 & $135-230$ & $169.95 \pm 27.50$ & & \\
\hline Group 3 & $136-252$ & $202.05 \pm 30.73$ & & \\
\hline Group 4 & 106-199 & $166.75 \pm 27.27$ & & \\
\hline \multicolumn{5}{|c|}{ Serum Ferritin $(n g / m l):$} \\
\hline Group 1 & $40-105$ & $74.05 \pm 20.15$ & 153.329 & $0.001 *$ \\
\hline Group 2 & $65-136$ & $106.65 \pm 22.78$ & & \\
\hline Group 3 & $250-647$ & $371.40 \pm 93.27$ & & \\
\hline Group 4 & $75-145$ & $108.75 \pm 19.11$ & & \\
\hline \multicolumn{5}{|l|}{ T. Sat (\%): } \\
\hline Group 1 & $22-93$ & $56.60 \pm 25.77$ & 17.427 & $0.001 *$ \\
\hline Group 2 & $20-52$ & $29.40 \pm 9.31$ & & \\
\hline Group 3 & $17-40$ & $29.75 \pm 6.02$ & & \\
\hline Group 4 & $26-39$ & $31.70 \pm 3.59$ & & \\
\hline \multicolumn{5}{|c|}{ S. Iron $(U g / m l)$ : } \\
\hline Group 1 & $0.4-1$ & $0.77 \pm 0.16$ & 0.143 & 0.934 \\
\hline Group 2 & $0.4-1$ & $0.77 \pm 0.16$ & & \\
\hline Group 3 & $0.4-1.1$ & $0.76 \pm 0.26$ & & \\
\hline Group 4 & $0.8-1.1$ & $0.79 \pm 0.21$ & & \\
\hline \multicolumn{5}{|c|}{$T I B C(m g / d l):$} \\
\hline Group 1 & $0.5-5.9$ & $3.10 \pm 1.40$ & 2.852 & $0.043 *$ \\
\hline Group 2 & $1.1-4.2$ & $3.09 \pm 0.94$ & & \\
\hline Group 3 & $1.4-3.1$ & $2.45 \pm 0.53$ & & \\
\hline Group 4 & $2.5-4.5$ & $3.19 \pm 0.65$ & & \\
\hline \multicolumn{5}{|c|}{ Hepcidin ferritin ratio: } \\
\hline Group 1 & $2-3.6$ & $2.63 \pm 0.45$ & 169.153 & $0.001 *$ \\
\hline Group 2 & $1.2-2.1$ & $1.59 \pm 0.26$ & & \\
\hline Group 3 & $0.2-0.8$ & $0.53 \pm 0.14$ & & \\
\hline Group 4 & $1.1-1.9$ & $1.52 \pm 0.24$ & & \\
\hline
\end{tabular}




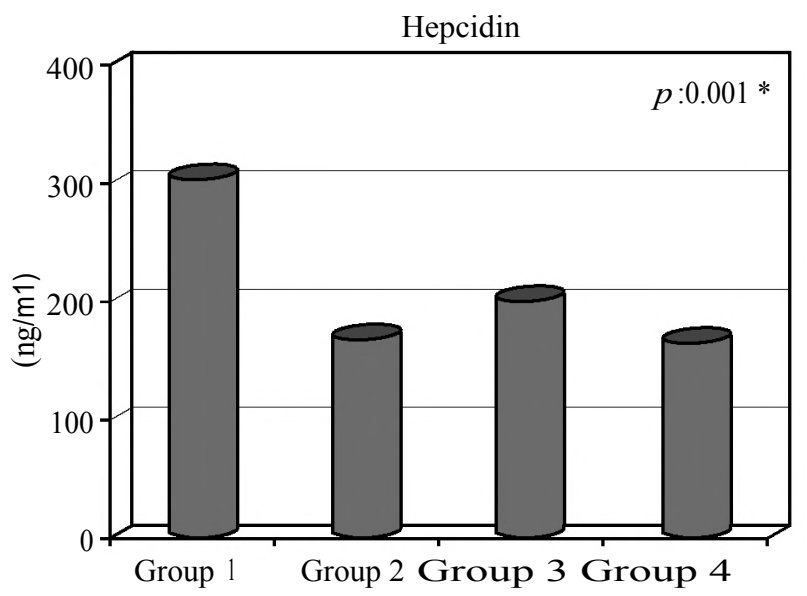

Fig. (1): Comparison between studied groups regarding Hepcidin

\section{Discussion}

Diabetes mellitus is a group of metabolic diseases characterized by hyperglycemia resulting from defects in insulin secretion or insulin action, or both. Diabetes and its complications have become a major public health problem in the world and its prevention has become a public health priority [5].

Iron is involved in binding and transporting oxygen, regulating cell growth and differentiation as well as electron transport, DNA synthesis and many important metabolic processes. Iron load is clearly linked to risk of developing T2D. It reduce B-cell insulin production and increase insulin resistance, it affect the metabolism of nutrient in skeletal muscle by decrease glucose oxidation and increase lipid oxidation, also it affect insulin receptors expression in hepatocyte [6]

Ferritin is also an acute-phase reactant, its synthesis is up-regulated by infection or inflammation. Studies have demonstrated that proinflammatory cytokines such as tumor necrosis factor (TNF)-a, interleukin- 1 (IL-1) and interleukin6 (IL-6) induce the expression of ferritin in cultured hepatic cell lines [7]. Type $2 \mathrm{DM}$ is closely correlated with chronic inflammation, increased circulating concentrations of IL-6 and TNF-a were found in type $2 \mathrm{DM}$. So, the increased ferritin levels in type $2 \mathrm{DM}$ probably also induced by their elevated inflammatory cytokines [8].

Systemic hepcidin is produced by hepatocyte and mainly regulated by iron load in the cells. Glucotoxicity inhibit glucose-stimulated insulin secretion and serum hepcidin expression and secretion which in turn reduce B-cell insulin synthesis

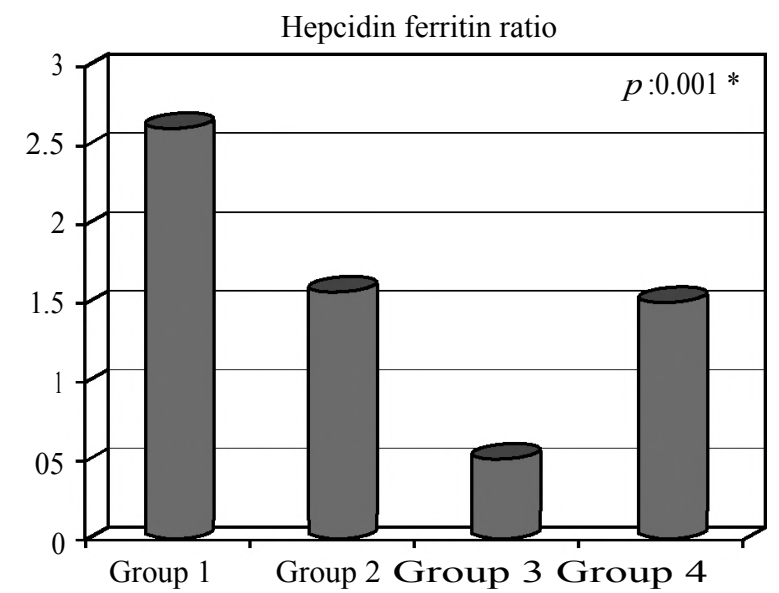

Fig. (2): Comparison between studied groups regarding hepcidin: Ferritin ratio

in T2D by inhibiting Pdx-1 expression, so transient overexpression in hepcidin was used to reverse the downregulation of Pdx-1 of insulin secretion induced by glucotoxicity [9]

This study consisted of randomized eighty subjects divided into four groups: Group 1: Included 20 patients with impaired glucose tolerance (prediabetes), Group 2: Included 20 patients with controlled diabetes, Group 3: 20 patients with uncontrolled diabetes, Group 4: Included 20 healthy volunteers.

This study was aimed to evaluate changes of serum hepcidin level in type 2 diabetes, assess possible relationships of serum hepcidin, iron status, hepcidin: Ferritin ratio and HOMA-IR in type 2 diabetes patients.

In these study, HOMA-IR was statistically higher in uncontrolled diabetes and lower in impaired glucose tolerance than controlled diabetes and healthy volunteers with $\left(p\right.$-value $\left.=0.007^{*}\right)$, this finding in agreement with Rajpathak et al., (2009) [1] who found that significant negative correlation between HOMA-IR, a surrogate of insulin resistance, and hepcidin: Ferritin ratio in impaired glucose tolerance with $\left(p\right.$-value $\left.=0.05^{*}\right)$, as iron is a strong pro-oxidant and high body iron levels are associated with increased level of oxidative stress that may elevate the risk of type 2 diabetes. Several epidemiological studies by You and Pickup et al., $[7,8]$ have reported a positive association between high body iron stores, as measured by circulating ferritin level, and the risk of type 2 diabetes and of other insulin resistant states such as the metabolic syndrome, gestational diabetes and polycystic ovarian syndrome. Results from these studies have evaluated the association between genetic mutations 
related to iron metabolism have been inconsistent and phlebotomy induced reduction in body iron levels may improve insulin sensitivity in humans. Also, this was coincided with results of Wang et al., (2014) [12] who found that Studies in rats have shown that insulin resistance can cause low hepcidin levels and that insulin therapy reversed these levels. These levels raised when insulin signaling was improved.

Serum hepcidin in our study was statistically highly significant in impaired glucose tolerance with $(p$-value $<0.001 *)$ than other studied groups and normal in controlled diabetes and uncontrolled diabetes and healthy volunteers, however the mean value of healthy volunteers was less than that of controlled diabetes and uncontrolled diabetes, this was coincided with results of Kulaksiz et al., (2008) [13] who found that hepcidin was statistically highly significant in impaired glucose tolerance with ( $p$ value $\left.<0.001^{*}\right)$, as Pancreatic beta cells were known to have the highest expression of hepcidin in pancreas tissue. Also, beta cells can co-secrete hepcidin and insulin, which further strengthened the links between glucose and iron metabolism. These mechanisms should be studied further to establish a better explanation of how insulin resistance affected hepcidin levels. In future studies, we intend to investigate the effect of insulin therapy on hepcidin levels by comparing these levels in different groups of patients with T2DM. But this was against the results of Simcox et al. (2013) [14] who found that hepcidin was statistically significantly low in risky type 2 diabetic subjects with $\left(p\right.$-value $\left.=0.636^{*}\right)$. Baseline serum hepcidin levels that were inadequately low in relation to body iron stores significantly predict the risk for T2DM, independently of a broad range of diabetes risk factors and determinants of hepcidin levels. Also, this was against with results of Sam et al., (2013) [15] who found that type 2 diabetes patients have significantly lower hepcidin with $\left(p\right.$-value $\left.=0.05^{*}\right)$ compared with weight-matched control. But Wang et al., (2014) [12] found that, there are two types of diabetic patients when it comes to hepcidin level; increase hepcidin level in T2D due to inflammatory process that occurs with T2D, and decrease hepcidin level is unclear, but insulin resistance is thought to play a role, so these suggests that regulation of insulin production could correct hepcidin level.

In our study, serum ferritin was statistically highly significant with $(p$-value $<0.001 *)$ in uncontrolled diabetes and lower in impaired glucose tolerance than controlled diabetes and healthy volunteers, This finding in agreement with You et al., (2005) [7] who found that serum ferritin con- centrations are significantly elevated in individuals with diabetes with $(p$-value $=0.0728 *)$ compared with the controls and were significantly correlated with risks of T2D. Ferritin is an acute-phase reactant and its synthesis is up-regulated by infection or inflammation. The chronic inflammatory state accompanied by T2D may also contribute to increased ferritin concentrations measured in T2D patients.

Hepcidin: Ferritin ratio in the our study was statistically highly significant in impaired glucose tolerance with $\left(p\right.$-value $\left.<0.001^{*}\right)$ and lower in uncontrolled diabetes than controlled diabetes and healthy volunteers, This finding in agreement with Sam et al., (2013) [15] who found that type 2 diabetes patients have significantly lower hepcidin: Ferritin ratio with $(p$-value $<0.001 *)$ compared with weight-matched control subjects. Reduced hepcidin: Ferritin ratio indicated inadequate hepcidin levels in response to the degree of iron overload. Reduced hepcidin concentrations may contribute to iron overload by increasing the intestinal absorption of iron. Moderately elevated iron levels were associated with elevated risk of type 2 diabetes. Also, Sam et al., (2013) [15] found that in impaired glucose tolerance, an inverse relationship between the hepcidin-ferritin ratio and insulin resistance with $\left(p\right.$-value $\left.=0.05^{*}\right)$, quantified by homeostasis model assessment-estimated insulin resistance, was detected, and iron depletion by phlebotomy was able to improve homeostasis model assessment estimated insulin resistance. Also, this was coincided with results of Rajpathak et al., (2009) [1] who found that hepcidin: Ferritin ratio was statistically higher in impaired glucose tolerance with ( $p$-value $\left.0.001^{*}\right)$ compared with weightmatched control subjects.

This study found that multifactorial effects on serum ferritin and hepcidin were found, so cannot be taken as markers for early diagnosis of type 2 diabetes, but hepcidin: Ferritin ratio is a meaningful parameter for early prediction of type 2 diabetes and can be used as a marker for insulin resistance and early diagnosis of impaired glucose tolerance (pre-diabetes).

\section{Conclusion:}

Serum hepcidin affected by multiple factors, so cannot be used for screening of type 2 diabetes. But hepcidin: Ferritin ratio could be a novel marker for early screening of patients with type 2 diabetes.

\section{Recommendations:}

Further studies are required to better understand the relationship between hepcidin, iron status, 
hepcidin: Ferritin ratio and HOMA-IR in type 2 diabetic patients.

Ongoing studies are needed to determine whether; hepcidin lowering agents may have a role in treating type 2 diabetes patients and correction of insulin signaling with insulin therapy could reverse hepcidin expression.

\section{Conflicts of interest:}

The authors declare no conflicts of interest.

\section{References}

1- RAJPATHAK S.N., CRANDALL J.P., WYLIE-ROSETT J., KABAT G.C., ROHAN T.E. and HU F.B.: The role of iron in type 2 diabetes in humans. Biochim Biophys Acta., 1790: 671-681, 2009.

2- NEMETH E., TUTTLE M.S., POWELSON J., VAUGHN M.B., DONOVAN A., WARD D.M., et al.: Hepcidin regulates cellular iron efflux by binding to ferroportin and inducing its internalization. Science, 306: 2090-2093, 2004.

3- VAN DIJK B.A., LAARAKKERS C.M., KLAVER S.M., JACOBS E.M., VAN TITS L.J., JANSSEN M.C., et al.: Serum hepcidin levels are innately low in HFE-related haemochromatosis but differ between C282Yhomozygotes with elevated and normal ferritin levels. Br. J. Haematol., 142: 979-985, 2008.

4- LE GUENNO G., CHANSEAUME E., RUIVARD M., MORIO B. and MAZUR A.: Study of iron metabolism disturbances in an animal model of insulin resistance. Diabetes. Res. Clin. Pract., 77: 363-370, 2007.

5- FERNANDEZ-REAL J.M., LOPEZ-BERMEJO A. and RICART W.: Cross-talk between iron metabolism and diabetes. Diabetes, 51: 2348-54, 2002.

6- PECHLANER R., WEISS G., BANSAL S., MAYR M., SANTER P., PALLHUBER B., et al.: Inadequate hepcidin serum concentrations predict T2D. Diabetes Metab. Res. Rev. Feb., 32 (2): 187-192, 2016.

7- YOU S.A. and WANG Q.: Ferritin in atherosclerosis. Clin. Chim. Acta., 357: 1-16, 2005.

8- PICKUP J.C., CHUSNEY G.D., THOMAS S.M. and BURT D.: Plasma interleukin-6, tumour necrosis factor alpha and blood cytokine production in type 2 diabetes. Life Sci., 67: 291-300, 2000.

9- MAO X., HUCHENG C., JUNMIN T., LIANGLIANG W. and TINGTING T.: Hepcidin links gluco-toxicity to pancreatic beta cell dysfunction by inhibiting Pdx-1 expression. Endocr. Connect., 6 (3): 121-128, 2017.

10-ASO Y., TAKEBAYASHI K., WAKABAYASHI S., MOMOBAYASHI A., SUGAWARA N., TERASAWA T., et al.: Relation between serum high molecular weight adiponectin and serum ferritin or prohepcidin in patients with type 2 diabetes. Diabetes. Res. Clin. Pract., 90: 250255,2010

11- FERNÁNDEZ-REAL J.M., EQUITANI F., MORENO J.M., MANCO M., ORTEGA F. and RICART W.: Study of circulating prohepcidin in association with insulin sensitivity and changing iron stores. J. Clin. Endocrinol. Metab., 94: 982-988, 2009.

12- WANG H., LI H., JIANG X., SHI W., SHEN Z. and LI M.: Hepcidin is directly regulated by insulin and plays an important role in iron overload in streptozotocininduced diabetic rats. Diabetes. May, 63 (5): 1506-1518, 2014.

13- KULAKSIZ H., FEIN E., REDECKER P., STREMMEL W., ADLER G. and CETIN Y.: Pancreatic beta-cells express hepcidin, an iron-uptake regulatory peptide. J. Endocrinol., 197 (2): 241-249, 2008.

14- SIMCOX J.A. and MCCLAIN D.A.: Iron and diabetes risk. Cell. Metab., 17 (3): 329-41, 2013.

15- SAM A.H., BUSBRIDGE M., AMIN A., WEBBER L., WHITE D., FRANKS S., et al.: Hepcidin levels in diabetes mellitus and polycystic ovary syndrome. Diabet. Med., 30 (12): 1495-9, 2013.

\section{تغيرات نسبة الهيبسيدين فى مرضى السكر من النوع الثانى}

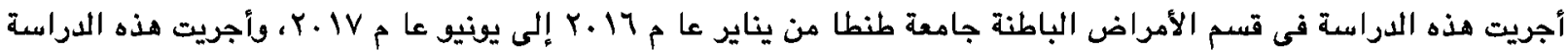

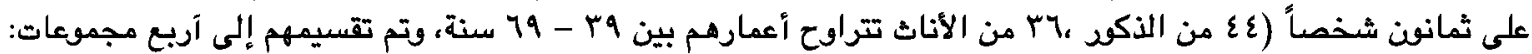

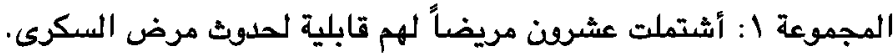

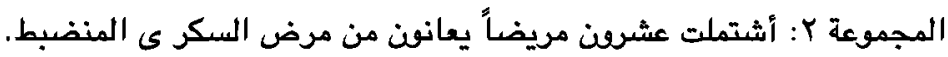

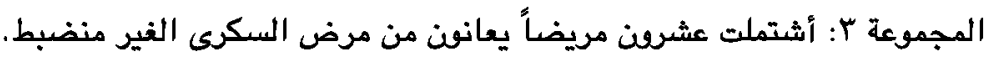

$$
\begin{aligned}
& \text { المجموعة ع: أشتملت عشرون شخصاً أصحاء كمجموعة ضابطة. }
\end{aligned}
$$

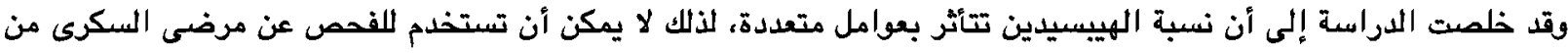

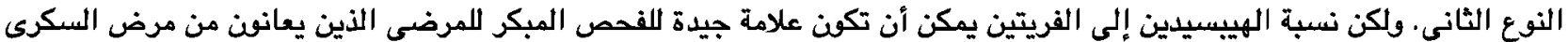

